\title{
Age-related differences in the quantitative analysis of the finger tapping task
}

\author{
Yu-Ri Kwon ${ }^{\mathrm{a}}$, Junghyuk Ko ${ }^{\mathrm{b}}$, Ryun-Hee Lee ${ }^{\mathrm{c}}$, Gwang-Moon Eom ${ }^{\mathrm{a}, \mathrm{c}}$ and Ji-Won Kim ${ }^{\mathrm{a}, \mathrm{c}, *}$ \\ ${ }^{a}$ BK21 Plus Research Institute of Biomedical Engineering, Konkuk University, Chungju, Korea \\ ${ }^{\mathrm{b}}$ Division of Mechanical Engineering, College of Engineering, Korea Maritime and Ocean University, \\ Busan, Korea \\ ${ }^{\mathrm{c}}$ Department of Biomedical Engineering, Konkuk University, Chungju, Korea
}

\begin{abstract}
.
BACKGROUND: Quantitative measures of the finger tapping task is important for objective assessment of bradykinesia. However, age-related changes in quantitative measures are still unclear.

OBJECTIVE: The aim of this study was to quantitatively investigate age-related group differences in finger tapping performance.

METHODS: Eighty-three healthy normal subjects with age ranging from aged 20 to 89 years participated in this study. All subjects were instructed to tap their index finger and thumbs as rapidly as possible and with as large amplitude as possible. Angular velocity of the finger tapping movement was measured using a gyrosensor. Quantitative variables include root mean squared (RMS) angular velocity, RMS angular displacement, peak power and peak frequency derived from angular velocity signals.

RESULTS: Significant age-related differences were observed in RMS angular velocity, peak power and peak frequency $(P<$ 0.001). Specifically, the oldest age group had the slowest average speed, the lowest peak power and peak frequency. These results indicate deterioration in finger speed, intensity of the main movement component and tapping frequency due to aging.

CONCLUSIONS: The results suggest that the quantitative variables should be adjusted for age when clinicians assess Parkinsonian bradykinesia. The results contribute to the development of an accurate and quantitative assessment tool for bradykinesia.
\end{abstract}

Keywords: Finger tapping, age, elderly, quantification, gyrosensor, Parkinson's disease

\section{Introduction}

The finger tapping task is one of the motor testing tools based on the unified Parkinson's disease rating scale (UPDRS) [1] and has been generally used to assess bradykinesia that in patients with Parkinson's disease (PD). Specifically, abnormalities associated with fine motor control have been assessed using the finger tapping task to detect the first signs of motor impairment in patients with PD [2]. However, the UPDRS relies on a clinician's subjective scoring ability based on clinical experience [3] and has poor resolution for the assessment of bradykinesia symptoms in early stages of PD [4]. Therefore, it is important to quantitatively measure finger tapping movements for an accurate diagnosis of disease progression and precise evaluation of treatment outcome.

\footnotetext{
${ }^{*}$ Corresponding author: Ji-Won Kim, Department of Biomedical Engineering, Konkuk University, 268 Chungwon-daero Chungju-si Chungcheongbuk-do 27478, Korea. Tel.: +82 43840 4953; Fax: +82 43851 0620; E-mail: kjw802@kku.ac.kr.
}

0928-7329 (c) 2022 - The authors. Published by IOS Press. This is an Open Access article distributed under the terms of the Creative Commons Attribution-NonCommercial License (CC BY-NC 4.0). 
Table 1

Subject demographics

\begin{tabular}{lcccc}
\hline \multirow{2}{*}{ Variables } & $\begin{array}{c}\text { Age group 1 } \\
(<30 \text { years, } n=20)\end{array}$ & $\begin{array}{c}\text { Age group 2 } \\
(30-49 \text { years, } n=17)\end{array}$ & $\begin{array}{c}\text { Age group 3 } \\
(50-70 \text { years, } n=22)\end{array}$ & $\begin{array}{c}\text { Age group 4 } \\
(>70 \text { years, } n=24)\end{array}$ \\
\cline { 2 - 5 } & Mean $(\mathrm{SD})$ & Mean $(\mathrm{SD})$ & Mean $(\mathrm{SD})$ & Mean $(\mathrm{SD})$ \\
\hline Age range & $20-27$ & $30-44$ & $50-69$ & $70-89$ \\
Age (years) & $22.25(2.35)$ & $33.59(3.70)$ & $60.36(5.37)$ & $78.75(5.83)$ \\
Height $(\mathrm{cm})$ & $168.32(7.95)$ & $169.94(8.19)$ & $157.00(7.13)$ & $155.29(7.99)$ \\
Weight $(\mathrm{kg})$ & $61.25(10.59)$ & $66.82(11.35)$ & $60.16(8.04)$ & $58.10(10.49)$ \\
BMI $\left(\mathrm{kg} / \mathrm{m}^{2}\right)$ & $21.35(2.63)$ & $23.00(2.61)$ & $24.32(3.01)$ & $23.92(2.87)$ \\
\hline
\end{tabular}

BMI, body mass index.

Many studies have investigated the quantification of the finger tapping task using inertial measurement unit (IMU) sensors. For example, gyro-sensors were used to quantitatively measure bradykinesia during the finger tapping task in patients with PD [5,6]. It has been reported that the average angular velocity, angular displacement, and frequency indices determined using a gyrosensor and they adequately reflected the characteristics of bradykinesia in PD [5,6]. Also, some studies suggested that magnetic sensors and accelerometers can be used in the quantitative assessment of the finger tapping task [7,8].

The deterioration in finger function with age leads to reduced quality of life. Indeed, elderly subjects often experience increased difficulties performing daily tasks [9]. PD is related to aging and affects nearly $1-2 \%$ of individuals aged 60 years and older [10]. However, it is difficult to distinguish PD-induced deterioration from aging-induced deterioration in finger tapping movements. It is important to distinguish the effects of age from those involving PD. Therefore, the investigation of age-matched normal features should precede an accurate assessment of upper-limb bradykinesia in patients with PD. Specifically, age-related changes in finger function should be investigated and normative data associated with finger tapping movements should be acquired. Although multiple studies have attempted to quantify finger tapping, few have investigated the age-related changes associated with the finger motor function. Some studies have performed a comparative analysis of hand function parameters such as hand grip force among young and elderly adults $[11,12]$. However, few quantitative measures of age-related changes involved the finger tapping task. Although AOKI et al. investigated finger tapping ability such as pinch force and tactile threshold, the study was limited by the small population sample and absence of standard clinical task (UPDRS). Therefore, our working hypothesis was that quantitative variables during the finger tapping motion deteriorate with aging. Accordingly, this study investigated age-related decline in finger tapping performance.

\section{Method}

\subsection{Subjects}

The present study included 83 healthy normal subjects ranging from 20 to 89 years of age. The subjects were classified into age group 1 ( $n=20$, age range 20-27 years), age group 2 ( $n=17$, age range 30 44 years), age group 3 ( $n=22$, age range 50-69 years) and age group 4 ( $n=24$, age range 70-89 years). All participants were recruited from local communities in Chungju city, Korea and provided written informed consent. Participants with any musculoskeletal disease or neurological disorders were excluded. Table 1 presents the participant demographics including age range, age, height, weight, and body mass index (BMI). 
Table 2

Differences in finger tapping performance between right and left fingers

\begin{tabular}{|c|c|c|c|}
\hline \multirow[t]{2}{*}{ Variables } & Right finger & Left finger & Significance \\
\hline & Mean (SD) & Mean (SD) & ( $p$-value) \\
\hline RMS angular velocity (deg/s) & $463.4(124.7)$ & $447.0(135.1)$ & 0.152 \\
\hline RMS angle (deg) & $23.3(6.9)$ & $23.3(7.6)$ & 0.942 \\
\hline Peak power $\left(\mathrm{deg}^{2} / \mathrm{s}^{2} / \mathrm{Hz}\right)$ & $104428427(79162471)$ & 96268658 (77952333) & 0.305 \\
\hline Peak frequency $(\mathrm{Hz})$ & $3.1(1.1)$ & $2.90(1.0)$ & 0.017 \\
\hline
\end{tabular}

\subsection{Experiments and analysis}

An independent measurement system based on a piezoelectric ceramic gyrosensor (CG-L53, NEC/Tokin, Japan) with small size $(6 \times 10 \times 25 \mathrm{~mm})$ and light weight $(0.26 \mathrm{~g})$ was used for measurement of angular velocity during finger tapping motions. The reliability of this system has been proved and the system has been described in previous studies $[5,6,13]$. The quantitative variables derived from the system strongly correlated with the clinical score. The gyrosensor was attached to the top of the index finger with the sensing axis aligned with finger flexion and extension. The finger tapping task was based on the finger tapping categories derived from UPDRS part III (motor examination). All subjects were instructed to tap their index finger and thumbs as rapidly as possible and with as large amplitude as possible. The angular velocity signals were recorded for $15 \mathrm{~s}$, which is the general test time for finger tapping task in the clinic. Both right and left fingers were measured once each. The sampling frequency of the recording was $250 \mathrm{~Hz}$.

To eliminate baseline drift and offset noise, a 4th-order zero phase digital high pass filter with a cutoff frequency of $1 \mathrm{~Hz}$ was used. Also, a low pass filter with a cutoff frequency of $50 \mathrm{~Hz}$ was used to eliminate high-frequency noise. The frequency range of the filters was determined via inspection of the power spectrum of the sensor signal $[5,6,13]$. As time domain variables, RMS (root mean squared) angular velocity and RMS angular displacement were derived from the gyrosensor signals. The angular displacement signal was derived from the numerical integration of the angular velocity. As frequency domain variables, peak power and peak frequency were derived from the power spectrum of the angular velocity signal. Age-related differences were evaluated by ANOVA (analysis of variance) and Tukey's post hoc test. Paired $t$-tests were performed to determine the differences between right and left fingers. The level of statistical significance was defined by $P<0.05$. All statistical analyses were performed using SPSS ver. 21 (SPSS Inc., USA)

\section{Results}

Figure 1 shows representative filtered angular velocity signals from the gyrosensor for each age group. Rhythmic angular velocity signals were observed by finger tapping motion. The amplitude of the angular velocity trajectories decreased with age.

As shown in Table 2, all variables except peak frequency were not significantly different between right and left fingers $(p>0.05)$. Although the peak frequency showed significant difference $(p<0.05)$, there was no interaction between finger and age factors $(p=0.96)$. These results indicate that the difference between right and left fingers had no affecting on age among all the variables.

Table 3 presents the results of ANOVA comparing the age groups. There were significant differences in all quantitative variables $(P<0.05)$ except RMS angular displacement $(P>0.05)$. As shown in Fig. 2 , significant age-related differences were found in RMS angular velocity. Specifically, the RMS angular 
Table 3

Results of ANOVA for four groups

\begin{tabular}{lccccc}
\hline \multirow{2}{*}{ Variables } & $\begin{array}{c}\text { Age group 1 } \\
(<30 \text { years, } \\
n=40)\end{array}$ & $\begin{array}{c}\text { Age group 2 } \\
(30-49 \text { years, } \\
n=34)\end{array}$ & $\begin{array}{c}\text { Age group 3 } \\
(50-70 \text { years, } \\
n=44)\end{array}$ & $\begin{array}{c}\text { Age group } 4 \\
(>70 \text { years, } \\
n=43)\end{array}$ & Significance \\
\cline { 2 - 6 } & Mean & Mean & Mean & Mean & $p$-value \\
\hline RMS angular velocity $(\mathrm{deg} / \mathrm{s})$ & 543.5 & 494.8 & 435.1 & 370.1 & 0.000 \\
RMS angle $(\mathrm{deg})$ & 23.6757 & 22.4782 & 24.3884 & 22.5239 & 0.572 \\
Peak power $\left(\mathrm{deg}^{2} / \mathrm{s}^{2} / \mathrm{Hz}\right)$ & 144716331.59 & 103416915.32 & 95792405.70 & 62091621.71 & 0.000 \\
Peak frequency $(\mathrm{Hz})$ & 3.53 & 3.57 & 2.69 & 2.36 & 0.000 \\
\hline
\end{tabular}

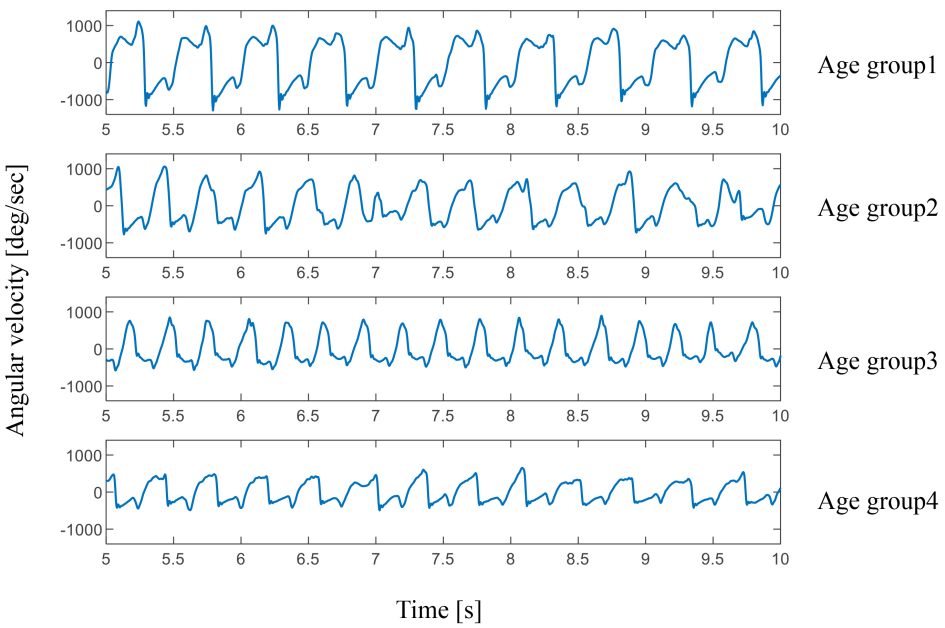

Fig. 1. Representative gyrosensor signals for each age group.

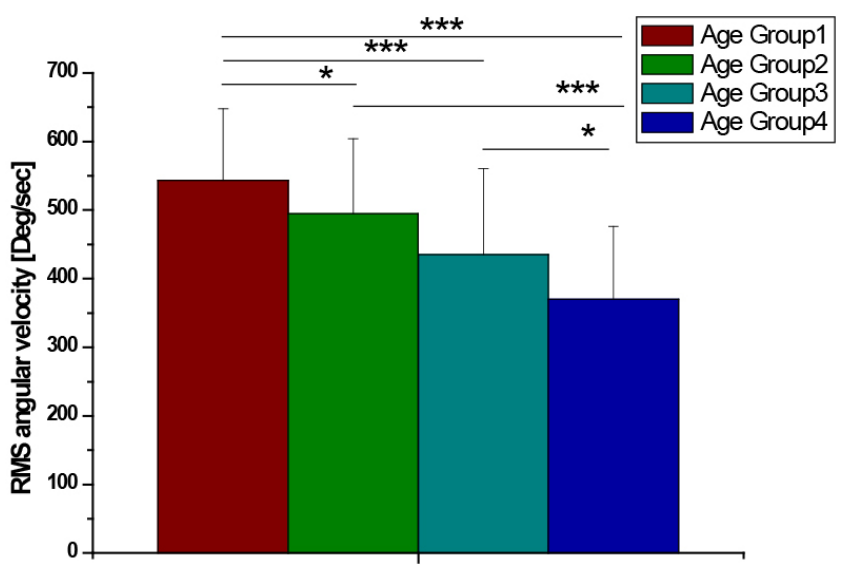

Fig. 2. Comparison of RMS angular velocity across different age groups $\left({ }^{*} p<0.05,{ }^{* * *} p<0.001\right)$.

velocity decreased with age. The RMS angular velocity of age group 4 was significantly lower than that of other age groups $(P<0.05)$. The RMS angular velocity of age group 1 was significantly greater than that of the other age groups $(P<0.05)$. In contrast, there was no significant difference in RMS angular displacement $(P>0.05)$ (Fig. 3). The peak power of age group 4 was significantly less than that of age 


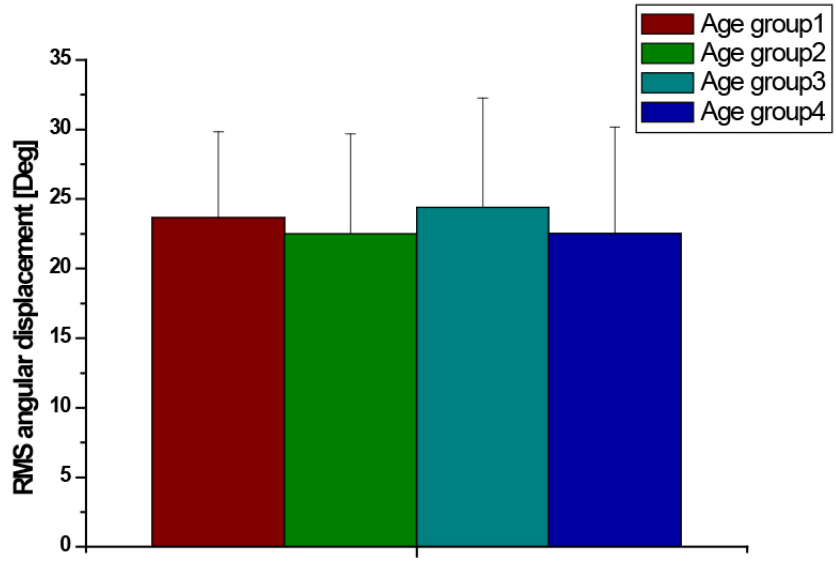

Fig. 3. Comparison of RMS angular displacement across different age groups.

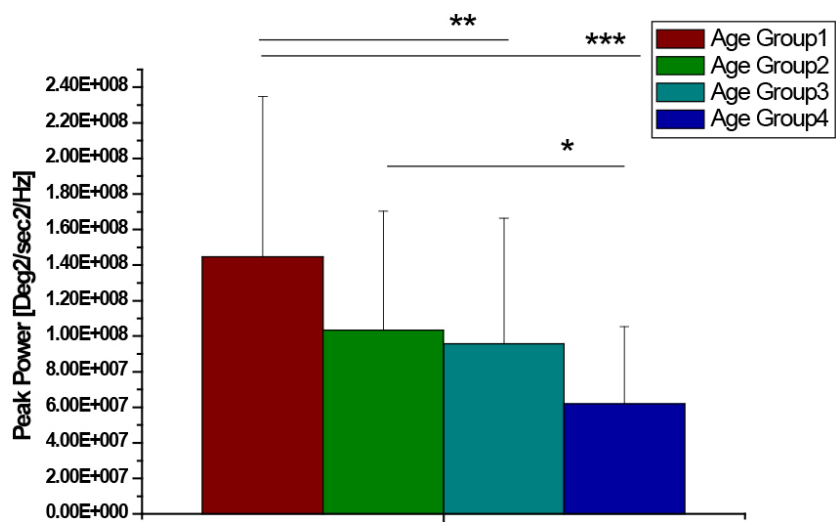

Fig. 4. Comparison of peak power between different age groups $\left({ }^{*} p<0.05,{ }^{* *} p<0.01,{ }^{* * *} p<0.001\right)$.

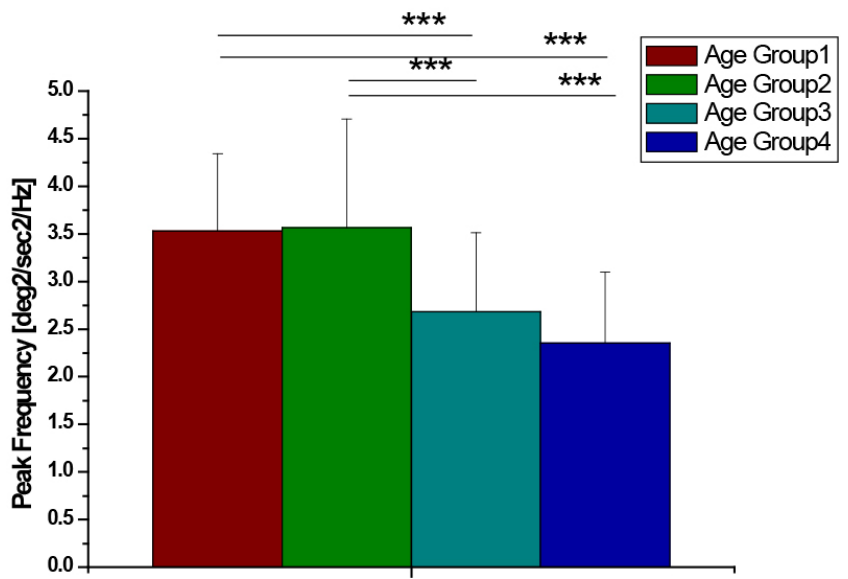

Fig. 5. Comparison of peak frequency between age groups $\left({ }^{* * *} p<0.001\right)$. 
groups 1 and $2(P<0.05)$ (Fig. 4). The peak power of age group 1 was significantly greater than that of age groups 3 and $4(P<0.01)$. Similar peak frequency was found between age groups 1 and 2 and between age groups 3 and 4 (Fig. 5). The peak frequencies of age groups 3 and 4 were significantly less than that of age groups 1 and 2 .

\section{Discussion}

In the present study, age-related differences in quantitative variables during finger tapping task were investigated. An age-related decline was observed in most quantitative variables. Specifically, the average speed of finger motion decreased with age, whereas the average amplitude of finger motion had no effect on age. Peak power of the angular velocity signals declined with age. Younger and older age groups showed lower peak frequency compared with young and middle age groups.

Finger tapping task is one of the clinical tools used to assess Parkinsonian bradykinesia. Recent studies quantified finger tapping motion using gyrosensors $[5,6]$ and accelerometers $[7,8]$. The studies demonstrated that quantitative measures of the IMU sensors are significantly correlated with clinical scores in finger tapping task. However, the studies failed to consider the possibility of age-related decline in quantitative variables. Distinguishing PD-induced deterioration of bradykinesia from aging-induced deterioration of bradykinesia is important for effective therapy and intervention in patients with PD. Further, adjustment for aging may increase the accuracy of bradykinesia assessment by improving the correlation coefficients between quantitative variables and clinical scores. The present study has demonstrated that some quantitative variables can differ with age.

In our study, the finger speed was decreased with age (Fig. 1). Especially, the oldest group had a slower average speed compared with the other age groups. This finding indicates that the finger speed deteriorated with aging. Further, a significant difference was found between age groups 3 and $4(P<$ $0.05)$. It has been reported that PD was increasingly prevalent in individuals aged 50 and older [15]. This indicates that age-related deterioration in the main age range of PD (age groups 3 and 4) should be analyzed further for an accurate assessment of bradykinesia. Although PD patients below age 50 were few relatively, their prevalence was 5.8 cases per 100,00 individuals [15]. Therefore, the effect of age should be considered in younger age groups as well as in older individuals. In contrast, the finger amplitude was not associated with aging, suggesting that only finger tapping speed depends on aging. This finding may be associated with the constant finger range of motion in most normal subjects regardless of finger speed. Bradykinesia is characterized by impaired speed and amplitude of movement [14]. Our results suggest that the speed factor should be adjusted for age during clinical evaluation of a patient with Parkinsonian bradykinesia. As described in a further study, a linear regression analysis based on age as independent variable is needed.

Frequency domain variables also showed significant age group differences. Age group 4 had the least peak power, whereas age group 1 had the greatest peak power, which indicates that the intensity of the main movement component of repetitive finger flexion and extension may deteriorate markedly in the oldest group. In contrast, groups 2 and 3 showed similar peak power. The peak frequency of older groups (age groups 3 and 4) was lower than that of younger groups (age groups 1 and 2), suggesting that the older group carried a higher main frequency component during finger tapping movement. Thus, the tap number per second was reduced with aging. However, the age-related decline in peak frequency differed when compared with finger speed, suggesting that the type of regression models used for adjustment of age should vary with each quantitative variable. 
A study found that finger tapping ability was worse in the elderly than in younger populations [16]. However, the study had limitations of small population and narrow age range. Our study measured finger tapping movements across a wide age range (four age groups) and demonstrated meaningful results suggesting that all quantitative variables except RMS angular displacement showed an age-related decline. Based on the results, quantitative variables excluding age may facilitate accurate clinical diagnosis of PD patients.

However, our study still has some limitations. First, no gender effect was considered along with quantitative variables analyzed in this study. Second, other clinical tasks such as forearm rotation and toe tapping may show age-related deterioration. Third, cognitive function may be associated with finger movement [17]. Therefore, a further study is required to investigate cognitive function as well as age and gender effects in many clinical tasks. Specifically, multiple regression models, including these factors, should be considered. Fourth, although this study recruited a higher number of subjects than the previous studies, additional validation using larger numbers of subjects in each age group is needed for the development of more accurate regression models.

\section{Conclusions}

Quantitative variables associated with finger motor function exhibited age-related declines. The average speed of finger tapping decreased significantly with age. In contrast, the amplitude of finger tapping was not affected by the age group. Frequency domain variables showed significant differences between specific age groups. The results contribute to the development of a regression model, which excludes the effect of age and leads to the development of a more accurate quantitative assessment tool for the selection of effective therapies for PD population.

\section{Acknowledgments}

This study was supported by the Basic Science Research Program through the National Research Foundation of Korea (NRF) funded by the Ministry of Education and Ministry of Science (2018R1C1B6008083).

\section{Conflict of interest}

None to report.

\section{References}

[1] Goetz CG, Tilley BC, Shaftman SR, Stebbins GT, Fahn S, Martinez-Martin P, et al. Movement disorder society-sponsored revision of the unified parkinson's disease rating scale (MDS-UPDRS): Scale presentation and clinimetric testing results. Mov Disord. 2008 Nov 15; 23(15): 2129-70.

[2] Taylor Tavares AL, Jefferis GS, Koop M, Hill BC, Hastie T, Heit G, et al. Quantitative measurements of alternating finger tapping in Parkinson's disease correlate with UPDRS motor disability and reveal the improvement in fine motor control from medication and deep brain stimulation. Mov Disord. 2005 Oct; 20(10): 1286-98.

[3] Machado A, Rezai AR, Kopell BH, Gross RE, Sharan AD, Benabid AL. Deep brain stimulation for Parkinson's disease: Surgical technique and perioperative management. Mov Disord. 2006 Jun; 21(Suppl 14): S247-58. 
[4] Koop MM, Shivitz N, Bronte-Stewart H. Quantitative measures of fine motor, limb, and postural bradykinesia in very early stage, untreated Parkinson's disease. Mov Disord. 2008 Jul 15; 23(9): 1262-8.

[5] Kim JW, Lee JH, Kwon Y, Kim CS, Eom GM, Koh SB, et al. Quantification of bradykinesia during clinical finger taps using a gyrosensor in patients with Parkinson's disease. Med Biol Eng Comput. 2010 Mar; 49(3): 365-71.

[6] Kim JW, Kwon Y, Yun JS, Heo JH, Eom GM, Tack GR, et al. Regression models for the quantification of Parkinsonian bradykinesia. Biomed Mater Eng. 2015; 26(Suppl 1): S2249-58.

[7] Kandori A, Yokoe M, Sakoda S, Abe K, Miyashita T, Oe H, et al. Quantitative magnetic detection of finger movements in patients with Parkinson's disease. Neurosci Res. 2004 Jun; 49(2): 253-60.

[8] Yokoe M, Okuno R, Hamasaki T, Kurachi Y, Akazawa K, Sakoda S. Opening velocity, a novel parameter, for finger tapping test in patients with Parkinson's disease. Parkinsonism Relat Disord. 2009 Jul; 15(6): 440-4.

[9] Shiffman LM. Effects of aging on adult hand function. Am J Occup Ther. 1992 Sep; 46(9): 785-92.

[10] Olanow CW, Stern MB, Sethi K. The scientific and clinical basis for the treatment of Parkinson disease (2009). Neurology. 2009 May 26; 72(21 Suppl 4): S1-136.

[11] Cole KJ. Grasp force control in older adults. J Mot Behav. 1991 Dec; 23(4): 251-8.

[12] Kinoshita H, Francis PR. A comparison of prehension force control in young and elderly individuals. Eur J Appl Physiol Occup Physiol. 1996; 74(5): 450-60.

[13] Kim JW, Kwon Y, Kim YM, Chung HY, Eom GM, Jun JH, et al. Analysis of lower limb bradykinesia in Parkinson's disease patients. Geriatr Gerontol Int. 2012 Apr; 12(2): 257-64.

[14] Espay AJ, Beaton DE, Morgante F, Gunraj CA, Lang AE, Chen R. Impairments of speed and amplitude of movement in Parkinson's disease: A pilot study. Mov Disord. 2009 May 15; 24(7): 1001-8.

[15] Park JH, Kim DH, Kwon DY, Choi M, Kim S, Jung JH, et al. Trends in the incidence and prevalence of Parkinson's disease in Korea: A nationwide, population-based study. BMC Geriatr. 2019 Nov 21; 19(1): 320.

[16] Aoki T, Fukuoka Y. Finger tapping ability in healthy elderly and young adults. Med Sci Sports Exerc. 2009 Mar; 42(3): 449-55.

[17] Rabinowitz I, Lavner Y. Association between finger tapping, attention, memory, and cognitive diagnosis in elderly patients. Percept Mot Skills. 2014 Aug; 119(1): 259-78. 